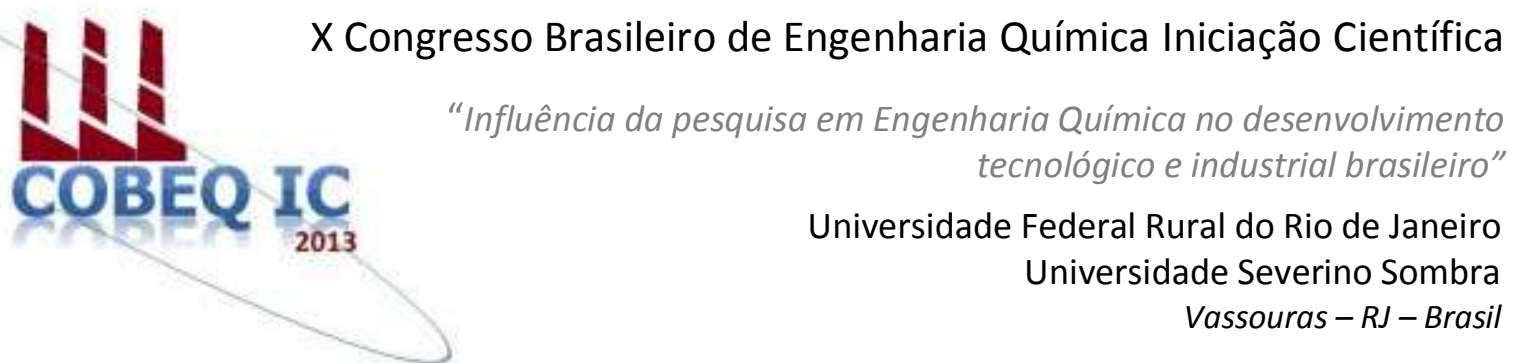

\title{
ANÁLISE DAS TAXAS DE EVAPORAÇÃO E EFICIÊNCIA TÉRMICA DO SECADOR DE LEITO DE JORRO
}

\author{
J. T. DIEB ${ }^{1}$, T. N. P. DANTAS ${ }^{2}$, F. C. MORAIS FILHO ${ }^{2}$, M. F. D. MEDEIROS ${ }^{3}$ \\ ${ }^{1}$ Aluno do DEQ/UFRN ${ }^{2}$ Mestrandos do DEQ/UFRN ${ }^{3}$ Professor do DEQ/UFRN \\ Departamento de Engenharia Química- Universidade Federal do Rio Grande do Norte \\ UFRN-Av. Senador Salgado Filho, 3000 Lagoa Nova, CEP.59078-970, Natal- RN \\ email: mariadefatima@eq.ufrn.br
}

\begin{abstract}
RESUMO - Para avaliação da capacidade de evaporação do secador de leito de jorro com partículas inertes empregado na secagem de pastas e alimentos líquidos, tais como polpas e suspensões de resíduos de frutas e leite, uma série de experimentos com alimentação contínua de água foram realizados seguindo um planejamento experimental fatorial do Tipo $2^{3} \mathrm{com}$ três réplicas no ponto central. As variáveis independentes foram a temperatura e vazão do ar e vazão de alimentação da água, sendo fixada uma carga de $2,5 \mathrm{~kg}$ de partículas de polietileno de alta densidade, empregado como material inerte. A partir dos balanços de massa e energia e das medidas de umidade relativa e temperaturas do ar e da parede acompanhadas ao longo de cada experimento, foram calculadas as taxas de evaporação e o calor gasto na evaporação da água, estimando-se as perdas de calor para o ambiente e a eficiência térmica do secador. Determinou-se o coeficiente convectivo de transferência de calor $h$, considerando a convecção natural entre a parede da coluna e o ar ambiente.
\end{abstract}

Palavras chave: coeficiente convectivo, perdas de calor, avaliação térmica.

\section{INTRODUÇÃO}

O secador leito de jorro com partículas inertes, empregado na secagem de pastas e suspensões, é considerado uma alternativa ao spray dryer visto que os produtos obtidos apresentam qualidade similar, a um custo menor. Os importantes resultados encontrados por Medeiros (2001), Medeiros et al. (2002), Medeiros et al. (2009), Souza et al. (2007a), Souza et al. (2007b), Rocha et al. (2011), com respeito a secagem de polpas de frutas mostram perspectivas bastante favoráveis à utilização do leito de jorro na secagem e produção de polpas de frutas em pó. Esse equipamento, além do baixo custo de aquisição, ainda apresenta algumas vantagens, dentre as quais se podem destacar as facilidades de construção, instalação e operação, o que permitiria sua implantação em pequenas agroindústrias ou cooperativas agrícolas, junto ao pequeno produtor rural, propiciando o processamento de suas frutas, produzidas na forma de pó.

Todavia, a viabilidade deste processo depende de uma série de fatores relativos à estabilidade fluidodinâmica do leito, consumo energético e ampliação de escala. Para melhor 
compreensão do comportamento térmico do leito é importante se conhecer a influência das variáveis operacionais sobre o desempenho do equipamento, no que se refere às taxas de evaporação e eficiência térmica do processo. A velocidade do gás na entrada é um parâmetro que influencia tanto a estabilidade quanto o desempenho térmico do equipamento, segundo Spitzener Neto (2001), o aumento da velocidade aumenta os coeficientes de transferência de calor e massa fluido-partícula e a energia de colisão diminuindo o acúmulo de sólidos do interior do jorro. A temperatura do gás na entrada também é um parâmetro de fundamental importância para $\mathrm{o}$ bom desempenho do secador estando, todavia, limitada pelas características e propriedades do material processado. Com relação à vazão da pasta, sabe-se que essa é uma das variáveis que controla a estabilidade fluidodinâmica do leito.Em muitos trabalhos são realizados experimentos de evaporação de água, considerada pasta padrão, para melhor compreensão do efeito das variáveis de operação sobre o comportamento do leito de jorro.

No presente trabalho foram realizados experimentos de evaporação de água no leito de jorro com partículas inertes de polietileno de alta densidade, sendo estimados, a partir dos balanços de massa e energia, as taxas de evaporação, o calor gasto na evaporação da água, as perdas de calor para o ambiente e a eficiência térmica do secador, analisando-se o efeito da vazão e temperatura do ar e da vazão da alimentação de água sobre o desempenho do processo.

\section{MATERIAIS E MÉTODOS}

\section{Equipamentos}

Os equipamentos utilizados para realização dos experimentos são mostrados na Figura 1. O módulo de secagem consta de uma coluna de aço inox e visores em acrílico, com $72 \mathrm{~cm}$ de altura e diâmetro de $18 \mathrm{~cm}$, com base cônica de $13 \mathrm{~cm}$ de altura e ângulo incluso de $60^{\circ}$. Um ciclone do tipo Lapple promove a separação e coleta do pó. Um soprador de $7 \mathrm{cv}$ fornece para o secador ar que é aquecido em um trocador de calor com resistência elétrica com potência de 2000 W. São utilizados termopares digitais para medidas de temperatura do ar na entrada e saída e em três pontos da parede da coluna. Manômetros de tubo em "U" para as tomadas de pressão. A vazão e umidade relativa do ar são medidas com anemômetro e termohigrômetro digitais. A alimentação contínua da água é realizada com bomba peristáltica conectada a um bico injetor de duplo fluido, em que o ar de atomização é fornecido por um compressor de baixa potência.

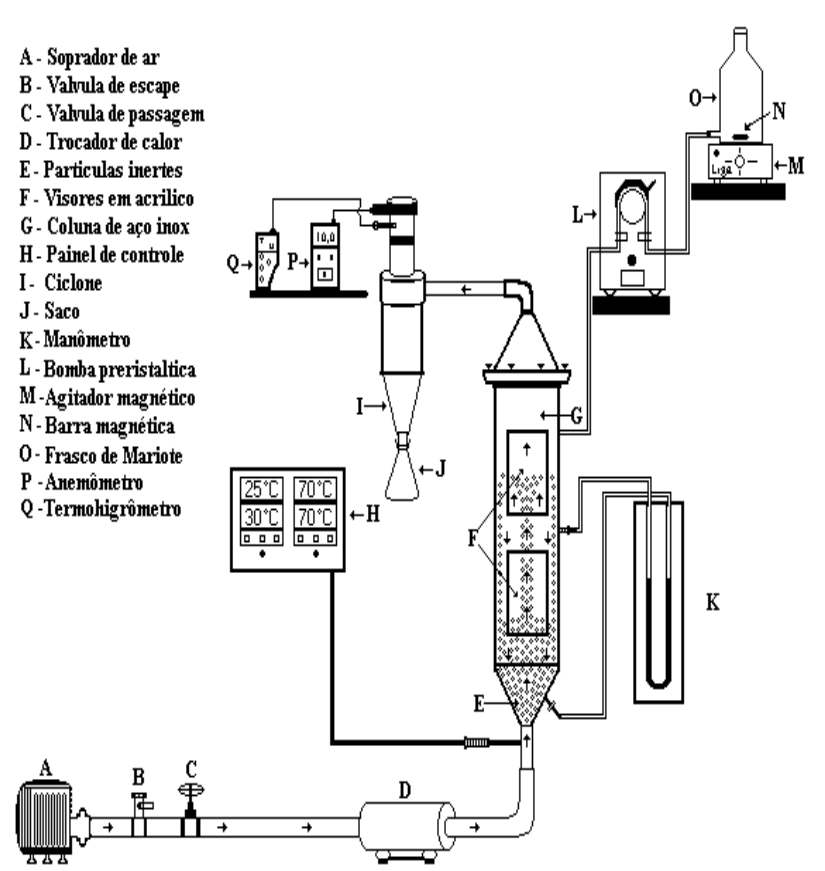

Figura 1 - Esquema do equipamento

\section{Metodologia}

Os experimentos foram realizados segundo um planejamento experimental do tipo fatorial $2^{3} \mathrm{com} 3$ réplicas no ponto central, totalizando 11 experimentos. Nas Tabelas $1 \mathrm{e}$ 2 são apresentados os níveis para as três variáveis, vazão de água e de ar e temperatura do ar na entrada do secador e as condições operacionais dos experimentos, respectivamente.

Tabela 1 - Níveis das variáveis.

\begin{tabular}{cccc}
\hline \multirow{2}{*}{ Nível } & \multicolumn{3}{c}{ Variável } \\
\cline { 2 - 4 } & $\mathrm{Q}_{\mathrm{p}}(\mathrm{mL} / \mathrm{min})$ & $\mathrm{W}_{\mathrm{ge}}(\mathrm{g} / \mathrm{s})$ & $\mathrm{T}_{\mathrm{ge}}\left({ }^{\circ} \mathrm{C}\right)$ \\
\hline-1 & 7 & 38,88 & 75 \\
\hline 0 & 15 & 42,41 & 95 \\
\hline+1 & 23 & 45,94 & 115 \\
\hline
\end{tabular}




\section{Tabela 2 - Condições de operação dos experimentos.}

\begin{tabular}{cccc}
\hline \multirow{2}{*}{ Experimento } & \multicolumn{3}{c}{ Nível } \\
\cline { 2 - 4 } & $\mathrm{Q}_{\mathrm{p}}{ }^{*}$ & $\mathrm{~W}_{\mathrm{ge}}{ }^{*}$ & $\mathrm{~T}_{\mathrm{ge}}{ }^{*}$ \\
\hline 01 & 0 & 0 & 0 \\
\hline 02 & 0 & 0 & 0 \\
\hline 03 & 0 & 0 & 0 \\
\hline 04 & 1 & -1 & -1 \\
\hline 05 & 1 & 1 & -1 \\
\hline 06 & -1 & -1 & -1 \\
\hline 07 & -1 & 1 & -1 \\
\hline 08 & 1 & -1 & 1 \\
\hline 09 & 1 & 1 & 1 \\
\hline 10 & -1 & -1 & 1 \\
\hline 11 & -1 & 1 & 1 \\
\hline
\end{tabular}

Partículas de polietileno de alta densidade (PEAD) foram utilizadas como material inerte, cujo diâmetro, densidade e esfericidade correspondem a $3,87 \times 10^{-3} \mathrm{~m}, 962$ $\mathrm{kg} / \mathrm{m}^{3}$ e 0,86 , respectivamente. Porosidade em leito estático de 0,29 e densidade aparente de $680 \mathrm{~kg} / \mathrm{m}^{3}$. Fixou-se uma carga de $2,5 \mathrm{~kg}$ de material inerte.

Metodologia de cálculo: Com os dados da umidade relativa e temperatura do ar na entrada e saída do secador, determinaram-se, através da carta psicrométrica as umidades absolutas, temperaturas de bulbo úmido $\mathrm{e}$ entalpias do ar.

As vazões de ar seco foram calculadas a partir da Equação 1 e com a Equação 2, que representa o balanço de ar seco, calcularam-se as taxas de evaporação.

$w_{s}=\frac{w_{g e}}{1+y_{g e}}=\frac{w_{g s}}{1+y_{g s}}$

$k=w_{s} \cdot\left(y_{g s}-y_{g e}\right)$

A partir do balanço de entalpia no secador, determinou-se, para cada experimento o calor perdido para o ambiente (Equação 3).

$$
w_{s} I_{g e}+w_{\mathrm{H}_{2} \mathrm{O}} \cdot c_{\mathrm{H}_{2} \mathrm{O}} \cdot T_{a m b}=w_{s} I_{g s}+Q_{p}
$$

Para cada intervalo de tempo considerado nos experimentos, foram estimados o calor latente de vaporização da água na temperatura de bulbo úmido do ar, calculando-se o calor gasto na evaporação através da Equação 4.

$$
Q_{e v}=k \cdot \lambda_{H_{2} O}^{T b u}
$$

A eficiência térmica foi definida como a razão entre o calor gasto na evaporação da água e o calor entregue pelo ar. (Equação 5).

$$
E_{f}=\left[\frac{Q_{e v}}{Q_{e v}+Q_{p}}\right] .100
$$

Os coeficientes convectivos foram determinados através da Equação 6.

$h=\frac{Q_{p}}{A\left(T_{p}-T_{a m b}\right)}$

Em todos os experimentos foram determinados os valores médios da taxa de secagem, do calor gasto na evaporação e do calor perdido para o ambiente, bem como a eficiência térmica e o coeficiente convectivo médio.

Para análise estatística dos efeitos das variáveis independentes $\left(\mathrm{T}_{\mathrm{ge}}, \mathrm{W}_{\mathrm{ge}} \mathrm{e} \mathrm{W}_{\text {água }}\right)$ sobre as variáveis respostas, taxa de secagem e eficiência térmica foi utilizado o programa Statistica 7.0.

\section{RESULTADOS E DISCUSSÕES}

Os resultados apresentados na Tabela 3 correspondem aos valores médios de todas as variáveis medidas e calculadas conforme a metodologia de cálculo descrita nos parágrafos anteriores: temperatura da parede e ambiente taxa de secagem, calor gasto na evaporação e perdido para o ambiente, eficiência térmica e coeficiente convectivo de transferência de calor. Também são exibidas as condições operacionais em que foram realizados os experimentos. 
Tabela 3 - Matriz experimental e resultados

\begin{tabular}{ccccccccccc}
\hline Exp & $\begin{array}{c}\mathrm{T}_{\mathrm{ge}} \\
\left({ }^{\circ} \mathrm{C}\right)\end{array}$ & $\mathrm{W}_{\mathrm{ge}}(\mathrm{g} / \mathrm{s})$ & $\mathrm{T}_{\mathrm{p}}\left({ }^{\circ} \mathrm{C}\right)$ & $\mathrm{T}_{\mathrm{amb}}\left({ }^{\circ} \mathrm{C}\right)$ & $\begin{array}{c}\mathrm{W}_{\text {água }} \\
(\mathrm{mL} / \mathrm{min})\end{array}$ & $\begin{array}{c}\mathrm{K} \\
(\mathrm{g} / \mathrm{s})\end{array}$ & $\begin{array}{c}\mathrm{Q}_{\mathrm{p}} \\
(\mathrm{W})\end{array}$ & $\begin{array}{c}\mathrm{Q}_{\mathrm{ev}} \\
(\mathrm{W})\end{array}$ & $\mathrm{h}_{\mathrm{p}}\left(\mathrm{W} / \mathrm{m}^{2} \mathrm{~K}\right)$ & $\operatorname{Ef}(\%)$ \\
\hline 1 & 95 & 42,41 & 49,2 & 30 & 15 & 0,2467 & 372,8 & 599,5 & 38,1 & 61,8 \\
\hline 2 & 95 & 42,41 & 48,8 & 30 & 15 & 0,2471 & 352,8 & 600,4 & 36,8 & 63,0 \\
\hline 3 & 95 & 42,41 & 48,6 & 30 & 15 & 0,2494 & 370 & 606,2 & 39,1 & 60,8 \\
\hline 4 & 75 & 38,88 & 39,3 & 28 & 23 & 0,3772 & 151,3 & 919,5 & 26,4 & 80,4 \\
\hline 5 & 75 & 45,94 & 37,6 & 29 & 23 & 0,3785 & 121,2 & 924,4 & 24,8 & 84,4 \\
\hline 6 & 75 & 38,88 & 47,4 & 29 & 7 & 0,1150 & 272,5 & 280,9 & 29,1 & 50,7 \\
\hline 7 & 75 & 45,94 & 52,2 & 28 & 7 & 0,1123 & 416,7 & 274,3 & 33,9 & 39,7 \\
\hline 8 & 115 & 38,88 & 52,6 & 29 & 23 & 0,3811 & 436,5 & 921,9 & 36,2 & 67,8 \\
\hline 9 & 115 & 45,94 & 52,0 & 29 & 23 & 0,3803 & 481,3 & 918,1 & 41,2 & 64,3 \\
\hline 10 & 115 & 38,88 & 67,7 & 29 & 7 & 0,1154 & 719,5 & 278,4 & 36,5 & 27,3 \\
\hline 11 & 115 & 45,94 & 63,4 & 29 & 7 & 0,1100 & 658,9 & 264,4 & 37,6 & 27,9 \\
\hline
\end{tabular}

Conforme se observa na Tabela 3 , a eficiência térmica do leito de jorro variou de $27,9 \%$ a $84,4 \%$ e sofreu importante influência da vazão de água alimentada demonstrando que em relação a capacidade térmica o equipamento foi subutilizado. Todavia, no experimento em que se atingiu a máxima eficiência, $\left(\mathrm{T}_{\mathrm{ge}}=75^{\circ} \mathrm{C}\right.$ e $\left.\mathrm{W}_{\text {água }}=23 \mathrm{~mL} / \mathrm{min}\right)$, ocorreram problemas de instabilidade fluidodinâmica do leito. Os coeficientes de transferência de calor foram mais baixos nos experimentos em que a vazão de água foi mais elevada com menores perdas de calor, conforme esperado. Apresentaram valores compreendidos numa faixa de 24,8 a 41,2 $\mathrm{W} / \mathrm{m}^{2} \mathrm{~K}$, mais elevados que os valores reportados na literatura para convecção livre do ar que variam de 2 a $25 \mathrm{~W} / \mathrm{m}^{2} \mathrm{~K}$ (Incropera \& De Wiu, 2008).

As taxas de evaporação sofreram variações no início do processo e tenderam a estabilizar ao longo do tempo como ilustram os gráficos exibidos nas Figuras 2 e 3 e 4.

Verifica-se, na Figura 2, a boa reprodutibilidade dos experimentos realizados nas condições do ponto central. As taxas de secagem estabilizaram em patamares compreendidos entre 0,19 e $0,23 \mathrm{~g} / \mathrm{s}$. As variações nas taxas iniciais são inerentes à fase transiente do processo.

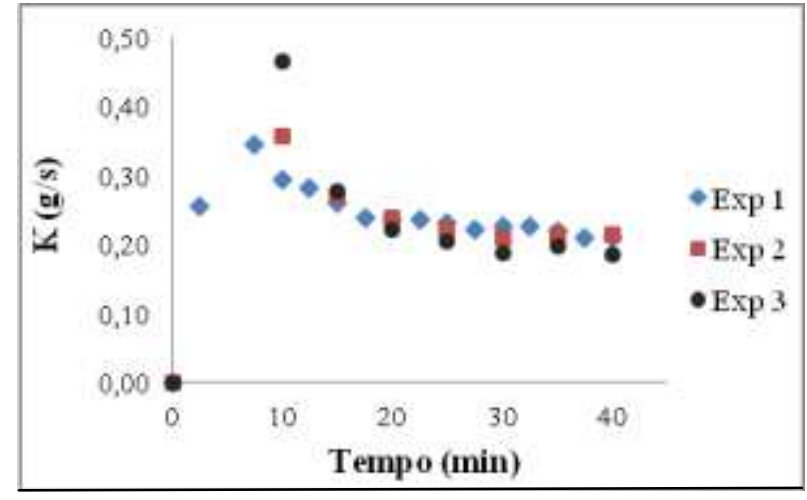

Figura 2 - Reprodutibilidade dos experimentos

Conforme se observa na Figura 3, mantendo-se fixas a vazão e temperatura do ar o aumento da vazão de água provocou importante aumento da taxa de evaporação. Fixando a temperatura e a vazão de água o aumento da vazão do ar não interferiu na taxa de evaporação.

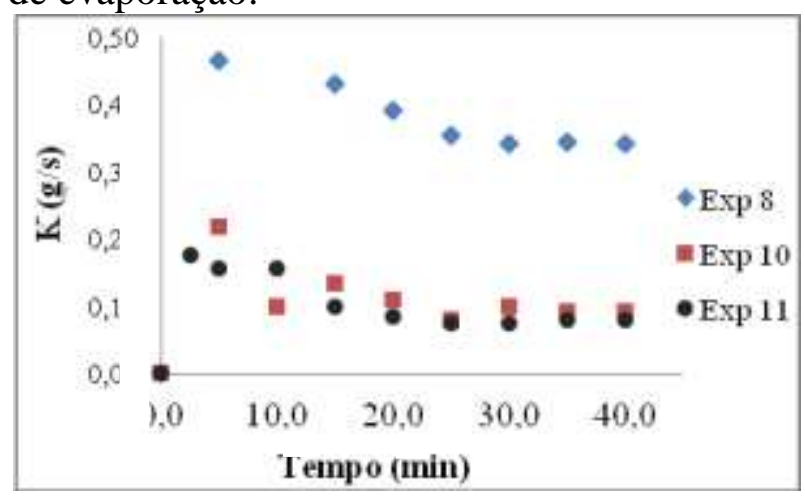

Figura 3 - Influência da vazão de alimentação de água e da vazão do ar sobre as taxas de evaporação. 


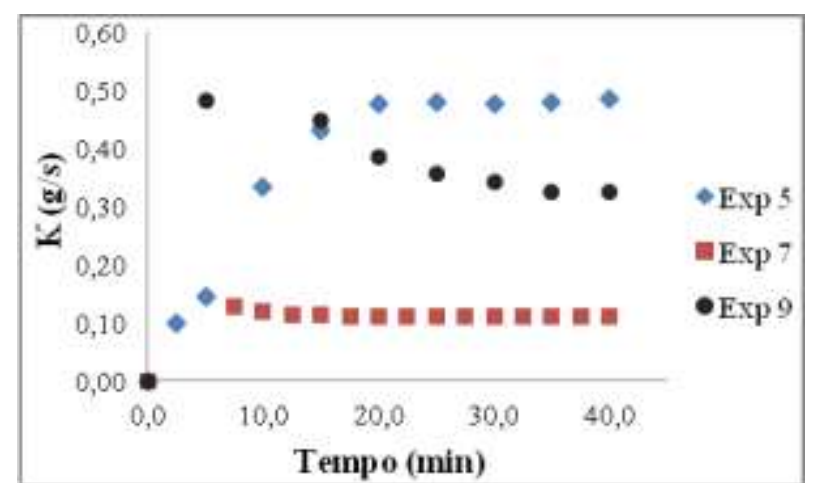

Figura 4 - Influência da vazão de alimentação de água e da temperatura do ar sobre as taxas de evaporação.

Na Figura 4 também se constata o efeito predominante da vazão de água sobre as taxas de evaporação, quando comparado com o efeito da temperatura. Para o experimento 7 com a temperatura fixada em $75^{\circ} \mathrm{C}$, a taxa de evaporação rapidamente se estabilizou em um patamar baixo e inferior ao observado no experimento 5 onde se manteve a mesma vazão e temperatura do ar, aumentando-se a vazão de alimentação. Ao se aumentar a temperatura para $115^{\circ} \mathrm{C}$, fixando-se as vazões de água e de ar (experimento 9), verifica-se que as taxas de evaporação iniciais foram elevadas mas tenderam a cair e se estabilizar em patamar mais baixo do que o observado no experimento 5 , com a temperatura fixada em $75^{\circ} \mathrm{C}$.

Os diagramas de Pareto ilustrados nas Figuras 5 e 6 exibem os efeitos das variáveis vazão de água, temperatura e vazão do ar sobre a taxa de secagem e a eficiência térmica, respectivamente.

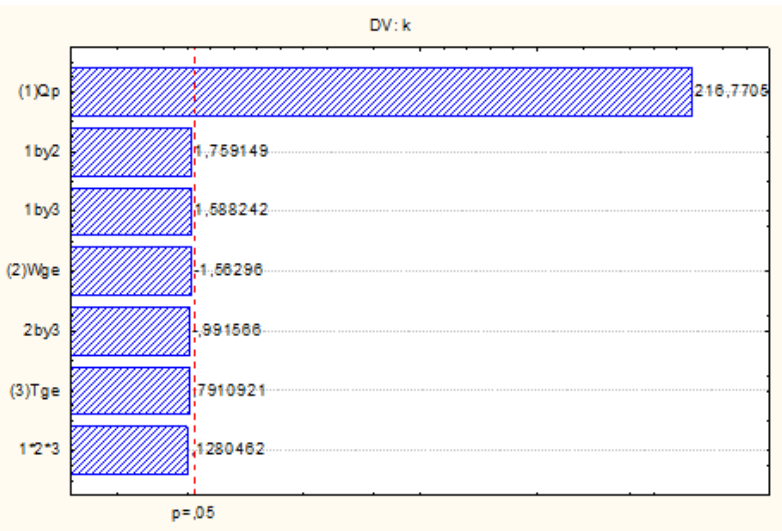

Figura 5 - Diagrama de Pareto (taxas de evaporação).

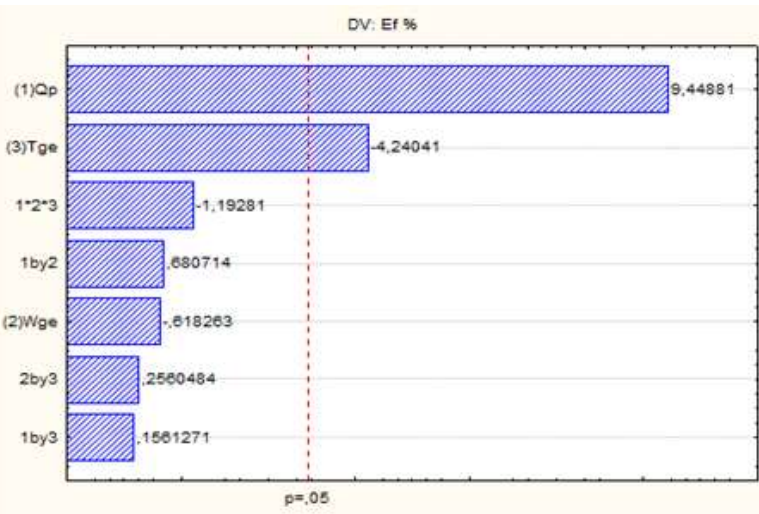

\section{Figura 6 - Diagrama de Pareto (Eficiência térmica).}

Conforme se observa na Figura 5, as taxas de evaporação sofreram importante influência da vazão de água, cujo efeito é positivo e significativo. As demais variáveis e interações entre as mesmas não apresentaram efeitos estatisticamente significativos, mas de baixa intensidade.

A vazão de água e a temperatura exerceram efeitos significativos e opostos sobre a eficiência térmica. Observa-se na Figura 6 que o efeito da vazão de água foi positivo e mais significativo do que o efeito da temperatura. Temperaturas do ar mais elevadas provocaram a diminuição da eficiência térmica.

\section{CONCLUSÕES}

Para a faixa de condições operacionais adotadas neste trabalho, a vazão do ar não influenciou a taxa de evaporação e a eficiência térmica. As taxas de evaporação sofreram variações no início do processo e tenderam a estabilizar ao longo do tempo. Foram independentes da vazão e temperatura do ar, mas influenciadas, significativamente, pela vazão da água. Os valores dos coeficientes convectivos calculados foram superiores aos valores reportados na literatura para convecção livre ar-parede. Todavia, o comportamento observado foi compatível com o fenomenologicamente esperado, pois aumentaram com a temperatura da parede e com as perdas de calor para o ambiente. A metodologia de cálculo levou a resultados consistentes, podendo ser adotada na modelagem do processo. 


\section{NOMENCLATURA}

A $\left(\mathrm{m}^{2}\right)$ - área da parede externa do secador considerando o corpo cilíndrico, a tampa e a base, ambas com configuração cônica;

$\mathrm{C}_{\text {água }}\left(\mathrm{J} / \mathrm{g}^{\circ} \mathrm{C}\right)-$ Calor específico da água;

Ef $(\%)$ - Eficiência térmica

$\mathrm{h}_{\mathrm{p}}\left(\mathrm{W} / \mathrm{m}^{2} \mathrm{~K}\right)$ - Coeficiente convectivo de trasferência de calor;

$\mathrm{I}_{\mathrm{ge}}(\mathrm{J} / \mathrm{g}$ de ar seco) - Entalpia específica do ar na entrada do secador;

$\mathrm{I}_{\mathrm{gs}}(\mathrm{J} / \mathrm{g}$ de ar seco) - Entalpia específica do ar na saída do secador;

$\mathrm{K}$ (g/s) - Taxa de evaporação;

$\mathrm{Q}_{\mathrm{ev}}(\mathrm{W})$ - Calor gasto na evaporação;

$\mathrm{Q}_{\mathrm{p}}(\mathrm{W})$ - Calor perdido para o ambiente;

$\mathrm{T}_{\mathrm{amb}}\left({ }^{\circ} \mathrm{C}\right)$ - Temperatura ambiente;

$\mathrm{T}_{\mathrm{ge}}\left({ }^{\circ} \mathrm{C}\right)$ - Temperatura do gás na entrada;

$\mathrm{T}_{\mathrm{p}}\left({ }^{\circ} \mathrm{C}\right)$-Temperatura na parede do secado;

$\mathrm{Tamb}\left({ }^{\circ} \mathrm{C}\right)$ - Temperatura ambiente;

$\mathrm{y}_{\mathrm{ge}}{ }^{-}$(g de água/g de ar seco) umidade absoluta do ar na entrada do secador;

$\mathrm{y}_{\mathrm{gs}^{-}}$(g de água/g de ar seco) umidade absoluta do ar na saída do secador;

$\mathrm{W}_{\text {água }}(\mathrm{ml} / \mathrm{min})$ - Vazão de água alimentada;

$\mathrm{W}_{\mathrm{ge}}(\mathrm{g} / \mathrm{s})$ - Vazão do ar na entrada do secador;

$\mathrm{W}_{\mathrm{gs}}(\mathrm{g} / \mathrm{s})$ - Vazão do ar na saída do secador;

$\mathrm{W}_{\mathrm{s}}$-Vazão do ar isento de umidade.

\section{REFERÊNCIAS}

INCROPERA, F. P. E; DEWITT, D. P. (2008), Fundamentos de Transferência de Calor e Massa. LTC Editora $6^{\text {a }}$ Ed., Rio de Janeiro.

MEDEIROS, M. F. D. Influência da composição química dos materiais no desempenho do processo de secagem de polpas de frutas em leito de jorro. Campinas: FEQ/UNICAMP, 2001. 247 p. Tese.

MEDEIROS, M. F. D., ROCHA, S. C. S., ALSINA, O. L. S., JERÔNIMO, C. E. M., MEDEIROS, U. K. L., MATA, A. L. M. L. (2002). "Drying of pulps of Tropical fruits in Spouted Bed: Effect of Composition on Dryer Performance. Drying Technology, v. 20, n. 4, p. 855881."

MEDEIROS, M. F. D.; SOUZA, J. S.; ALSINA, O. L. S.; ROCHA, S. C. S. (2009), Drying of tropical fruit pulps: an alternative spouted bed process. In: Maria Laura Passos; Cláudio P. Ribeiro. (Org.). Innovation in Food Engineering. New York: CRC Press - Taylor \& Francis Group, p. 361-388

ROCHA, S. C. S.; Souza, J. S.; ALSINA, O. L. S.; MEDEIROS, M. F. D. (2011). Drying of Tropical Fruit Pulps: Spouted Bed Process Optimization as a Function of Pulp Composition. Drying Technology, 2011, v. 29, p. 1587-1599.

SOUZA, J. S.; ARAUJO, V. P. U. ; SOUZA JR., F. E. ; PEDRINI, M. R. S. ; ROCHA, S. C. S. ; MEDEIROS, M. F. D. (2007a). Estudo comparativo das propriedades físicas e físico-químicas de um mix in natura e o pó reconstituído. XLVII Congresso Brasileiro de Química, 2007, Natal. Anais do XLVII Congresso Brasileiro de Química, 2007.

SOUZA, J. S.; ARAUJO, V. P. U. ; ROCHA, S. C. S.; SOUZA JR., F. E. ; MEDEIROS, M. F. D. (2007b). Estudo higroscópico e caracterização do mix em pó obtido através do processo de secagem em Leito de Jorro. In: XLVII Congresso Brasileiro de Química, 2007, Natal/RN. Anais do XLVII Congresso Brasileiro de Química, 2007

SPITZER NETO, P. I., CUNHA, F. O., FREIRE, J. T. (2001), The influence of the paste feed in the minimum spouting velocity. Braz. J. Chem. Eng. 18, 243-251.

\section{AGRADECIMENTOS}

Ao $\mathrm{CNPq}$ e à Propesq, pelo apoio financeiro. 